\title{
High Pressure Behaviour of Elastic and Mechanical Properties of NiGa Intermetallic Compound
}

\author{
F. ZEGRAR ${ }^{a}$, S. BOUCETtA ${ }^{b, *}$ AND B. OTHMANI ${ }^{b}$ \\ ${ }^{a}$ Ferhat Abbas-Sétif 1 University, Department of Physics, Setif, Algeria \\ ${ }^{b}$ Ferhat Abbas-Sétif 1 University, Department of E.B.T., Setif, Algeria
}

\begin{abstract}
We have employed the density functional theory plane-wave pseudopotential method with local density approximation and generalized gradient approximation to investigate the structural, elastic and mechanical properties of the intermetallic compound NiGa. The calculated equilibrium lattice constant and bulk modulus are in good agreement with the experimental and other calculated values. According to our best knowledge, from the elastic constants, the bulk modulus $B$, anisotropy factor $A$, shear modulus $G$, the Young modulus $E$ and the Poisson ratio $\sigma$ for $\mathrm{NiGa}$ compound are obtained for the first time. By comparison, our results for the elastic constants $C_{i j}$, bulk modulus $B$, shear modulus $G$ and the Young modulus $E$ are as good as those of NiAl compound. The dependences of the elastic and mechanical parameters of NiGa compound on pressure were also investigated. It was found that the cubic NiGa compound is mechanically stable under pressure according to the elastic stability criteria up to $13 \mathrm{GPa}$, and it is not elastically isotropic. By analyzing the ratio $(B / G)$, it was concluded that NiGa compound is ductile in nature.
\end{abstract}

DOI: 10.12693/APhysPolA.130.471

PACS/topics: 71.20.Lp, 62.20.-x, 62.50.-p

\section{Introduction}

Many investigations on the $\mathrm{Ni}-\mathrm{Ga}$ phase diagram have been performed and many intermetallic compounds were reported in this system $(\mathrm{Ni}-\mathrm{Ga})$ such as $\alpha^{\prime}-\mathrm{Ni}_{3} \mathrm{Ga}, \beta$ $\mathrm{NiGa}, \gamma-\mathrm{Ni}_{13} \mathrm{Ga}_{7}, \delta-\mathrm{Ni}_{5} \mathrm{Ga}_{3}$ and $\varepsilon-\mathrm{Ni}_{3} \mathrm{Ga}_{2}$ [1-3]. In the last years, intermetallics were of particular interest, both from the point of view of their fundamental properties and their practical applications as materials with excellent chemical, mechanical and physical properties. Some intermetallics are interesting functional materials, others have attracted attention as structural materials for hightemperature applications [4]. Literature survey reveals that the NiGa intermetallic compound is less reported.

In order to fully take advantage of the properties of NiGa intermetallic compound for more technological applications, theoretical explorations of the structural, elastic and mechanical properties are necessary. In this paper, therefore, we present a systematic study on the equilibrium structure, elastic constants, and mechanical parameters of NiGa compound. Then the elastic constants were calculated under pressure and the mechanical stability was evaluated by using first-principles calculations based on density functional theory. The rest of the paper is organized as follows: the computational method is given in Sect. 2. The numerical results and discussion are given in Sect. 3, and finally a conclusion is presented in Sect. 4.

\section{Computational method}

In this study, first-principles calculations are performed with the plane-wave pseudopotential (PW-PP)

\footnotetext{
*corresponding author; e-mail: sd.boucetta@gmail.com
}

total energy method as implemented in the Cambridge Serial Total Energy Package (CASTEP) simulation program [5]. This is based on the density functional theory (DFT) $[6,7]$. The approximations: local density approximation (LDA) developed by Ceperley and Adler and parameterized by Perdew and Zunger [8, 9], as well as the generalized gradient approximation (GGA) with the new functional of the Perdew-Burke-Ernzerhof (PBE), known as PBE for solids (PBEsol) [10], are made for electronic exchange-correlation potential energy. The Coulomb potential energy caused by electron-ion interaction is described using the Vanderbilt-type ultra-soft scheme [11], in which the orbitals of $\mathrm{Ni}\left(3 d^{8} 4 s^{2}\right)$, Ga $\left(3 d^{10} 4 s^{2} 4 p^{1}\right)$, are treated as valence electrons. The cutoff energy for the plane-wave expansion was $300 \mathrm{eV}$ and the Brillouin zone sampling was carried out using the $10 \times 10 \times 10$ set of the Monkhorst-Pack mesh [12]. The structural parameter $(a)$ of NiGa was determined using the Broyden-Fletcher-Goldfarb-Shenno (BFGS) minimization technique [13]. This method usually provides the fast way of finding the lowest energy structure.

In the structural optimization process, the energy change, maximum force, maximum stress and maximum displacement are set as $1.0 \times 10^{-5} \mathrm{eV} /$ atom, $0.03 \mathrm{eV} / \AA$, $0.05 \mathrm{GPa}$, and $0.001 \AA$, respectively.

\section{Results and discussion 3.1. Structural properties}

The atomic structure of NiGa compound crystallizes in a cubic lattice of CsCl-type structure with the space group Pm-3m (221) and the equilibrium lattice parameter has a value of $2.886 \AA$ [14]. The initial unit cell structural model of the NiGa compound is built according to the experimental data, where the nickel atom $(\mathrm{Ni})$ is positioned at $(0,0,0)$ and the gallium $(\mathrm{Ga})$ atom is positioned at $(1 / 2,1 / 2,1 / 2)$ [14], as shown in Fig. 1. 


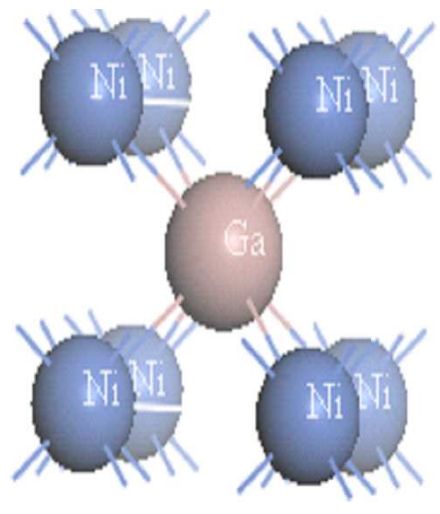

Fig. 1. The cubic structure of NiGa compound.

The results of calculated lattice parameter $a$ of NiGa compound using the PP-PW method within both the LDA and the GGA approximations are listed in Table I, along with the available experimental and theoretical data. From Table I, the calculated lattice constant $a$ is only $0.87 \%$ smaller than the experimental value using LDA and it is only $0.31 \%$ higher than the experimental value using the GGA-PBEsol. Our calculated equilibrium lattice parameter $a$ is in excellent agreement with the experimental data and it is quite equal to the experimental value in Ref. [15] from GGA-PBEsol calculation.

TABLE I

Calculated and experimental values of the equilibrium lattice constant $a[\AA]$ in cubic NiGa compound.

\begin{tabular}{cc|c}
\hline \hline This work: & LDA & 2.861 \\
& GGA & 2.895 \\
\hline Exp. & {$[14]$} & 2.886 \\
& {$[15]$} & 2.895 \\
\hline Calc. & {$[15]$} & 2.923
\end{tabular}

\subsection{Elastic and mechanical properties}

The elastic constants were determined from a linear fit of the calculated stress-strain function according to the Hooke law [16]. The cubic crystal has three independent elastic constants, $C_{11}, C_{12}$ and $C_{44}$. The bulk modulus $B$ is related to the elastic constants by

$$
B=\frac{C_{11}+2 C_{12}}{3} \text {. }
$$

In Table II, the calculated elastic constants and the bulk modulus of NiGa at zero pressure are presented.

To the best of our knowledge, there are no experimental and other theoretical data in literature for the elastic constants $\left(C_{i j}\right)$ of $\mathrm{NiGa}$ for comparison, so we consider the present results as prediction study which still awaits an experimental confirmation. We have included in Table II some experimental data of NiAl compound for comparison [17]. The $C_{11}, C_{12}, C_{44}$ and $B$ obtained within GGA and LDA are much closer to the experimental values of $\mathrm{NiAl}$.

\section{TABLE II}

Calculated values of the elastic constants $C_{i j}[\mathrm{GPa}]$, bulk modulus $B$ [GPa], anisotropy factor $A$, shear modulus $G[\mathrm{GPa}]$, the Young modulus $E[\mathrm{GPa}]$, the Poisson ratio $\sigma$ and $B / G$ in cubic NiGa compound, and experimental values of $\mathrm{NiAl}[17]$.

\begin{tabular}{c|c|c|c|c|c|c|c|c|c}
\hline \hline & $C_{11}$ & $C_{12}$ & $C_{44}$ & $B$ & $A$ & $G$ & $E$ & $\sigma$ & $B / G$ \\
\hline LDA & 208.57 & 163.58 & 89.94 & 178.57 & 3.99 & 51.93 & 142.02 & 0.37 & 3.44 \\
GGA & 171.59 & 145.56 & 84.19 & 154.24 & 6.46 & 41.07 & 113.17 & 0.38 & 3.75 \\
\hline NiAl & 199 & 137 & 116 & 158 & & & & &
\end{tabular}

The Zener anisotropy factor $A$ of NiGa compound is calculated by the following equation [18]:

$$
A=\frac{2 C_{44}}{C_{11}-C_{12}} \text {. }
$$

As shown in Table II, the calculated Zener anisotropy factor is larger than 1 , which indicates that this compound is higher elastic anisotropic. The elastic constants $C_{i j}$ are estimated for monocrystal NiGa. However, the prepared materials are in general polycrystalline, and therefore it is important to evaluate the corresponding moduli for the polycrystalline phase. For this purpose we have applied the Voigt-Reuss-Hill (VRH) approximation [19-21]. For the cubic system, the Reuss and Voigt bounds on the shear modulus are given by

$$
\begin{aligned}
G_{R} & =\frac{5 C_{44}\left(C_{11}-C_{12}\right)}{4 C_{44}+3\left(C_{11}-C_{12}\right)}, \\
G_{V} & =\frac{1}{5} C_{11}-C_{12}+3 C_{44} .
\end{aligned}
$$

Finally, the VRH mean value is obtained by

$$
G=\frac{1}{2}\left(G_{V}+G_{R}\right) \text {. }
$$

We also calculated the Young modulus $E$ and the Poisson ratio $\sigma$ which are frequently measured for polycrystalline materials when investigating their hardness. These quantities are related to the bulk modulus and the shear modulus by the following equations [22]:

$$
\begin{gathered}
E=\frac{9 B G}{3 B+G}, \\
\sigma=\frac{3 B-E}{6 B} .
\end{gathered}
$$

The shear modulus $G$, the Young modulus $E$ and the Poisson ratio $\sigma$ for NiGa compound, calculated from the elastic constants are also listed in Table II. The results demonstrate that the $\mathrm{NiGa}$ intermetallic compound is stiffer in terms of bulk, the Young and shear modulus. The ratio $B / G$ is a simple relationship related to brittle or ductile behaviour of materials; it has been proposed by Pugh [23]. A high $B / G$ ratio is associated with ductility, whereas a low value corresponds to the brittleness. The critical value separating ductile and brittle material is 1.75. The calculated results are listed in Table II. The results indicate that $\mathrm{NiGa}$ can be classified as ductile material at zero pressure.

\subsection{High pressure behaviour of NiGa compound}

In order to investigate the high pressure behaviour of NiGa compound, we have optimized the structural pa- 
rameter of its cubic lattice at different pressure up to $20 \mathrm{GPa}$. As a very good agreement between experimental data and calculated results, we still used GGA approximation in this study. We next evaluate the dependence of the elastic constants on hydrostatic pressure. In Fig. 2, we present the variation of the elastic constants $\left(C_{11}, C_{12}\right.$ and $C_{44}$ ) and the bulk modulus $B$ of NiGa with respect to the variation of pressure from 0 to $20 \mathrm{GPa}$.

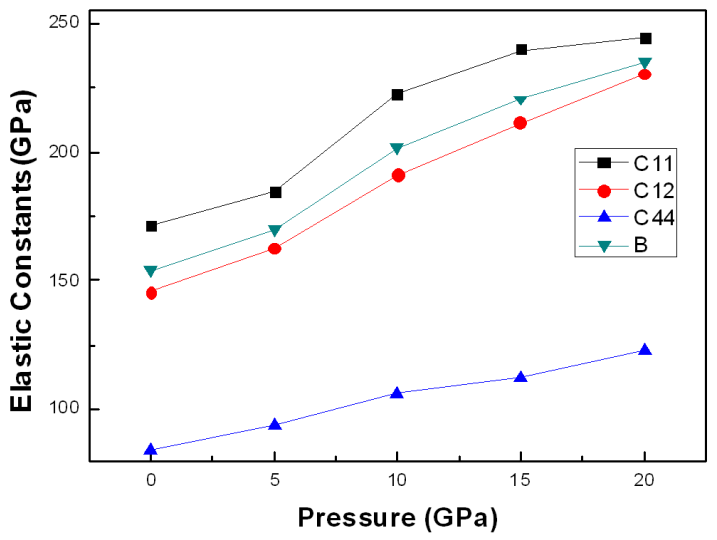

Fig. 2. GGA pressure dependence of the elastic constants $C_{11}, C_{12}$ and $C_{44}$ and the bulk modulus $B$ for NiGa compound.

We observe a linear dependence in all curves in the considered range of pressure. It is easy to observe that the elastic constants $C_{11}, C_{12}, C_{44}$ and the bulk modulus $B$ increase when the pressure is enhanced in this compound. The $C_{11}$ is the most sensitive constant to the hydrostatic pressure, whereas $C_{44}$ is the least sensitive one.

For a cubic crystal under pressure $P$, the generalized elastic stability criteria $[24,25]$ are

$$
K=C_{11}+2 C_{12}+P>0, \quad K_{1}=C_{44}-P>0
$$

and

$$
K_{2}=C_{11}-C_{12}-2 P>0 .
$$

The obtained results are depicted in Fig. 3. As shown in Fig. 3, these criteria are satisfied only when the pressure is smaller than $15 \mathrm{GPa}$. Consequently this compound is mechanically stable up to $13 \mathrm{GPa}$ and it is unstable mechanically above 13 GPa. Please use different $-\mathrm{y}$ axis for the $K$.

The Zener anisotropy factor $A$ of NiGa compound is calculated at various pressures and the results are shown in Table III. As a result in Table III, it can be seen that NiGa compound exhibits highest elastic anisotropy with increasing pressure. The shear modulus $G$, and the Young modulus $E$ of $\mathrm{NiGa}$ at various pressures are also calculated and the results are listed in Table III. It can be seen from Table III that the pressure affects significantly the $G$ and $E$ moduli. The results indicate that the values of $G$ and $E$ increase with the increase of pressure. It is interesting to point out that the NiGa compound has a strongest mechanical behaviour. We also studied

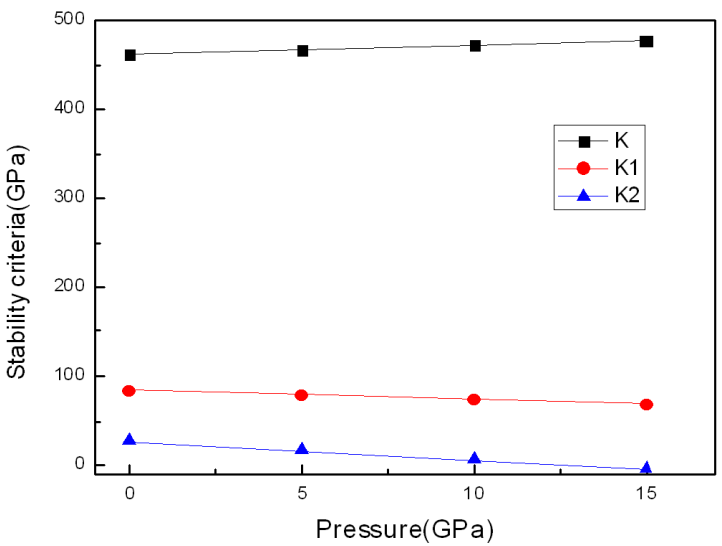

Fig. 3. Stability criteria for NiGa vs. pressure.

the effect of pressure on the $B / G$ ratios and the results are listed in Table III. It can be seen that the $B / G$ ratios increase with an increase in pressure. As shown in Table III, it is clear that NiGa compound remains in a ductile manner and it has larger ductility under high pressure than ambient pressure.

TABLE III

The elastic constants $C_{i j}$ [GPa], bulk modulus $B$ [GPa], anisotropy factor $A$, shear modulus $G[\mathrm{GPa}]$, and the Young modulus $E[\mathrm{GPa}]$ at different pressures $P[\mathrm{GPa}]$.

\begin{tabular}{c|c|c|c|c|c|c|c|c}
\hline \hline$P$ & $C_{11}$ & $C_{12}$ & $C_{44}$ & $B$ & $A$ & $G$ & $E$ & $B / G$ \\
\hline 0 & 171.59 & 145.56 & 84.19 & 154.24 & 6.47 & 41.07 & 113.17 & 3.75 \\
5 & 184.25 & 162.49 & 93.65 & 169.75 & 8.61 & 41.85 & 116.02 & 4.06 \\
10 & 222.52 & 191.05 & 106.37 & 201.54 & 6.71 & 51.16 & 141.51 & 3.94 \\
15 & 239.63 & 211.08 & 112.22 & 220.60 & 7.86 & 51.51 & 143.37 & 4.28
\end{tabular}

\section{Conclusion}

In this paper, the ground state properties as well as the high pressure behaviour of NiGa intermetallic compound have been studied by means of the density functional theory plane-wave pseudopotential method, within the LDA and GGA. The calculated equilibrium lattice parameter and bulk modulus of NiGa compound are in good agreement with the available experimental and theoretical data. By comparison, it is found that the GGA calculated results of elastic constants $C_{11}, C_{12}, C_{44}$, and the bulk modulus $B$ are as good as those for the NiAl experimental values. The computed results of the born stability criteria indicate that NiGa compound is mechanically stable up to $13 \mathrm{GPa}$. It is found that the Zener factor $A$ suggests that NiGa compound exhibits high elastic anisotropy at various pressures. The isotropic bulk modulus $B$, shear modulus $G$ and the Young modulus $E$ of the NiGa compound were determined using the VRH averaging scheme. The results indicate that NiGa has the strongest mechanical behaviour. It is found that the $\mathrm{NiGa}$ compound remains in ductile nature in the entire pressure range studied. Our results suggest there are 
potential technological applications of such material in extreme environments.

\section{References}

[1] S.Y. Lee, P. Nash, Phase Diagrams of Binary Nickel Alloys, ASM International, Materials Park (OH) 1991, p. 133.

[2] W.B. Pearson, D.M. Rimek, Can. J. Phys. 35, 1228 (1957).

[3] R. Ducher, R. Kainuma, K. Ishida, J. Intermet. 15, 148 (2007).

[4] J.H. Westbrook, R.L. Fleischer, Intermetallic Compounds - Principles and Practices), Wiley, New York 1995.

[5] M.D. Segall, P.J.D. Lindan, M.J. Probert, C.J. Pickard, P.J. Hasnip, S.J. Clark, M.C. Payne, J. Phys. Condens. Matter 14, 2717 (2002).

[6] P. Hohenberg, W. Kohn, Phys. Rev. B 136, 864 (1964).

[7] W. Kohn, L.J. Sham, Phys. Rev. A 140, 113 (1965).

[8] D.M. Ceperley, B.J. Alder, Phys. Rev. Lett. 45, 566 (1980).

[9] J.P. Perdew, A. Zunger, Phys. Rev. B 23, 5048 (1981).

[10] J.P. Perdew, A. Ruzsinszky, G.I. Csonka, O.A. Vydrov, G.E. Scuseria, L.A. Constantin, X. Zhou, K. Burke, Phys. Rev. Lett. 100, 136406 (2008).
[11] D. Vanderbilt, Phys. Rev. B 41, 7892 (1990).

[12] H.J. Monkhorst, J.D. Pack, Phys. Rev. B 13, 5188 (1976).

[13] T.H. Fischer, J. Almlof, J. Phys. Chem. 96, 9768 (1992).

[14] R. Guerin, A. Guivarch, J. Appl. Phys. 66, 2122 (1989).

[15] Li-Shing Hsu, Y.-K. Wang, G.Y. Guo, J. Appl. Phys. 92, 1419 (2002).

[16] J.F. Nye, Physical Properties of Materials, Dunod, Paris 1961, (in French).

[17] A.V. Ponomareva, E.I. Isaev, Yu.Kh. Vekilov, I.A. Abrikosov, Phys. Rev. B 85, 144117 (2012).

[18] C. Zener, Elasticity and Anelasticity of Metals, University of Chicago Press, Chicago 1948.

[19] W. Voigt, Handbook of Crystal Physics, Terubner, Leipzig 1928, (in German).

[20] A. Reuss, Z. Angew. Math. Mech. 9, 49 (1929).

[21] R. Hill, Proc. Phys. Soc. Lond. Sect. A 65, 349 (1952).

[22] E. Schreiber, O.L. Anderson, N. Soga, Elastic Constants and Their Measurements, McGraw-Hill, New York 1973.

[23] S.F. Pugh, Philos. Mag. 45, 823 (1954).

[24] S. Yip, J. Li, M. Tang, J. Wang, Mater. Sci. Eng. A 317, 236 (2001)

[25] G.V. Sin'ko, A. Smimov, J. Phys. Condens. Matter 14, 6989 (2002). 